\title{
Changes in the PD-1 and PD-L1 expressions of splenic dendritic cells in multiple-organ dysfunction syndrome mice and their significance
}

\author{
Q. Liu, J.Y. Lu, X.H. Wang, B.J. Qu, S.R. Li and J.R. Kang \\ Department of Pathology, \\ The First Affiliated Hospital of General Hospital of PLA, Beijing, China \\ Corresponding author: J.Y. Lu \\ E-mail: jylucn@126.com
}

Genet. Mol. Res. 13 (3): 7666-7672 (2014)

Received June 25, 2013

Accepted July 2, 2014

Published September 26, 2014

DOI http://dx.doi.org/10.4238/2014.September.26.4

\begin{abstract}
The aim of this study was to evaluate the expression of surface molecules in splenic dendritic cells (DC) in multipleorgan dysfunction syndrome (MODS) mice and their effects on the immunosuppression of sepsis and MODS. One hundred thirty C57BL/6 mice were divided into 7 groups: 6, 12, 24, 48 h, 5-7 days, 10-12 days, and the normal control group. The sepsis-MODS mouse model was established by zymson injection into the peritoneal cavity. Histopathological changes in the spleen were evaluated by hematoxylin and eosin (HE) staining. After enrichment with $\mathrm{BD}^{\mathrm{TM}}$ IMag, the expressions of PD-1, PD-L1, MHC-II (I-A $\left.{ }^{b}\right)$, and CD86 in splenic DCs were examined by flow cytometry, and their relationship with sepsis development and MODS was analyzed. The histological structures of the spleen were damaged in the 24-, 48-h, and 10-12day groups. PD-L1 expression increased $6 \mathrm{~h}$ after zymosan injection, decreased to normal levels at 24 and $48 \mathrm{~h}$, and increased at 5-7 days, peaking at 10-12 days. The change in PD-1 expression roughly
\end{abstract}


paralleled that of PD-L1. MHC-II and CD86 increased at 6 and $12 \mathrm{~h}$, and dropped to normal levels at 10-12 days. In the early stage of injury, splenic DCs were mainly activated, whereas in the later stage, the expressions of the negative co-stimulatory molecules, PD-L1 and PD1 , were upregulated, similar to tolerogenic DCs. Splenic DCs might suppress the stimulation of T lymphocytes in MODS mice through the PD-L1/PD-1 pathway, which would induce immunosuppression and the pathogenesis of MODS.

Key words: Multiple organ failure; Dendritic cells; PD-1/PD-L1; Spleen

\section{INTRODUCTION}

Pyemia and multiple-organ dysfunction syndrome (MODS) are important causes of death in surgical intensive care unit patients. Studies have shown that pyemia can induce apparent changes in the body's inflammation-immune system. Early non-specific inflammatory cells are activated, and the body manifests excessive inflammatory reactions. Furthermore, $\mathrm{T}$ and B lymphocytes show a high degree of apoptosis, residual lymphocytes present hypofunction, and the body manifests immune hypofunction and even immunosuppression. Although the majority of patients with pyemia can withstand early inflammatory reactions, a considerable portion of such patients dies of immunosuppression-caused uncontrollable infections. Therefore, the study of the immune system is very relevant for the prevention and treatment of pyemia and MODS, especially with respect to the immunosuppression occurrence mechanism (Lyn-Kew and Standiford, 2008). At present, the pyemia immunosuppression occurrence mechanism is still unclear.

The function of $\mathrm{T}$ lymphocytes depends on the activity of antigen-presenting cells, and dendritic cells (DCs) are a special type of antigen-presenting cell. DCs can specifically stimulate and regulate the immune response of $\mathrm{T}$ cells. They not only induce the immune response of T cells, but can also induce immunosuppression. Studies have shown that DCs can induce initial T cell proliferation, inhibit activated T cells, and promote $\mathrm{T}$ cell apoptosis by means of negative co-stimulatory molecules (Selenko-Gebauer et al., 2003; Keir et al., 2008). Programmed cell death receptor-1 (PD-1) and its ligand (PD-L1) are newly described negative co-stimulatory signal molecule members of the B7 family (Freeman et al., 2000; Nishimura and Honjo, 2001; Tseng et al., 2001). The DCs of patients with human immunodeficiency virus (HIV), hepatitis B virus (HBV), and tumors were found to inhibit the T cell immune response via the PD-L1/PD-1 pathway (Xu et al., 2010; Peng et al., 2011; Brahmer et al., 2012).

We hypothesized that pyemia could cause the increase of PD-L1 and PD-1 expressions in DCs, and that DCs participate in suppression of the body's T cell immune function via the PD-L1/PD-1 pathway. This study evaluated changes in the expressions of splenic DC PD-1 and PD-L1 molecules of MODS mice during the disease course of pyemia-MODS, and investigated the roles of the PD-1/PD-L1 inhibition pathway in immune tolerance and immunosuppression in the advanced stage of pyemia in order to seek a new regulation target point for MODS prevention and treatment. 


\section{MATERIAL AND METHODS}

\section{Experimental animals and grouping}

A total of 130 male C57BL/ 6 mice, with body weight ranging from 20 to $25 \mathrm{~g}$ and age ranging from 6 to 8 weeks, were purchased from the Experimental Animal Center of the Military Medical Science Academy. Mice were randomly divided into the control group (10 mice) and the test group (120 mice). The test group was further divided into the 6-, 12-, 24-, 48-h, 5-7-day, and 10-12-day subgroups after zymosan-induced trauma (20 mice in each subgroup).

\section{Model preparation}

The MODS model was established in mice in the test group in the following manner: $1 \mathrm{~g}$ zymosan powder and $40 \mathrm{~mL}$ medical liquid paraffin wax were mixed and prepared into a $25 \mathrm{~g} / \mathrm{L}$ zymosan suspension. The zymosan suspension was sterilized in a $100^{\circ} \mathrm{C}$ water bath for $80 \mathrm{~min}$ and then cooled to room temperature. After the abdominal skin was sterilized, $0.6 \mathrm{mg} / \mathrm{g}$ zymosan suspension was administered by intraperitoneal injection. Sampling was conducted by killing live mice at corresponding time points. In addition, the mice in the normal control group were conventionally fed, and sampling was randomly conducted by killing live mice.

\section{Pathological examination}

For all mice, the spleen was fixed with $4 \%$ formaldehyde, paraffin wax sections were conventionally prepared, and observations were conducted under a light microscope.

\section{Cell suspension preparation}

After $500 \mu \mathrm{L}$ collagenase $\mathrm{D}$ was injected into the spleen, the spleen tissue was shorn and incubated at $37^{\circ} \mathrm{C}$ for $25 \mathrm{~min}$. Subsequently, $10 \mathrm{mM}$ ethylenediaminetetraacetic acid (EDTA) was added, and the spleen tissue was incubated again for 5 min and ground in a 400mesh metal sieve with a grinding rod to collect the cell suspension. Next, the cell suspension was centrifuged at $1000 \mathrm{rpm}$ for $10 \mathrm{~min}$. The collected cells were suspended in $2 \mathrm{~mL}$ phosphate-buffered saline (PBS), and diamine blue staining was conducted. The resultant solution was then smeared onto blood cell counting plates to observe the viable cell count (it should be over 95\%) under a light microscope; the cell concentration was adjusted to $1 \times 10^{6}$ cells $/ \mathrm{mL}$.

\section{DC enrichment}

Five microliters biotinylated DC-enrichment "cocktail" (CD2, CD3e, CD45R, CD49b, CD147, LY-6G, TER-119) was added to $10^{6}$ cells and incubated for $15 \mathrm{~min}$ on ice. Next, the resultant mixture was washed with 10 time volumes $1 \mathrm{X}$ BD magnetic bead buffer and centrifuged at $1000 \mathrm{rpm}$ for $7 \mathrm{~min}$ to remove the supernatant. Subsequently, $5 \mu \mathrm{L}$ magnetic beads $/ 10^{6}$ cells was added into the deposition and mixed well. The mixture was incubated at $6^{\circ}-12^{\circ} \mathrm{C}$ for $30 \mathrm{~min}$, and the cells were transferred into a round-bottom detection tube (the maximum volume was $1 \mathrm{~mL}$ ). The detection tube was placed on the magnet frame for 6-8 
min, and the supernatant was drawn into a new tube for detection. Flow cytometry was used to evaluate the separating effect.

\section{Flow cytometry}

For flow cytometry, $0.5 \mu \mathrm{L}$ antibodies FITC-CD11c+PE-PD-1, FITC-CD11c+PE-PDL1, FITC-CD11c+PE-CD86, and PE-CD11c+FITC-I-A ${ }^{\mathrm{b}}$ were respectively added into four detection tubes and mixed well. For each detection tube, the mixture was placed at $4{ }^{\circ} \mathrm{C}$ for 30 min, washed with 0.1 M PBS three times, and detected with a flow cytometer. In addition, the negative control and the mono-antibody control were established for each time of detection.

\section{Statistical analysis}

The SPSS 10 statistical analysis software was used. Experimental data are reported as means \pm standard deviation (means $\pm \mathrm{SD}$ ), and the Student $t$-test was used for the statistical analysis of data. $\mathrm{P}<0.05$ was considered to be a significant difference.

\section{RESULTS}

\section{Pathological morphology observations}

In the 6- and 12-h groups, white pulp lymphocytes were dense and plentiful, the acini lienalis proliferated, and the sinus lienis was dilated and congested. In the 24- and 48-h groups, the white pulp area was reduced, the acini lienalis number was reduced, periarterial lymphatic sheath lymphocytes were reduced, and the boundary of the red and white pulp was unclear. In addition, neutrophilic granulocyte infiltration was visible. In the 5-7-day group, the white pulp was reduced, lymphocytes were sparse, and the acini lienalis was reduced. In the 1012-day group, the white pulp was obviously reduced, and the periarterial lymphatic sheath disappeared and was accompanied by more neutrophilic granulocyte infiltration. Furthermore, hemorrhage was visible in a few cases (Figure 1).

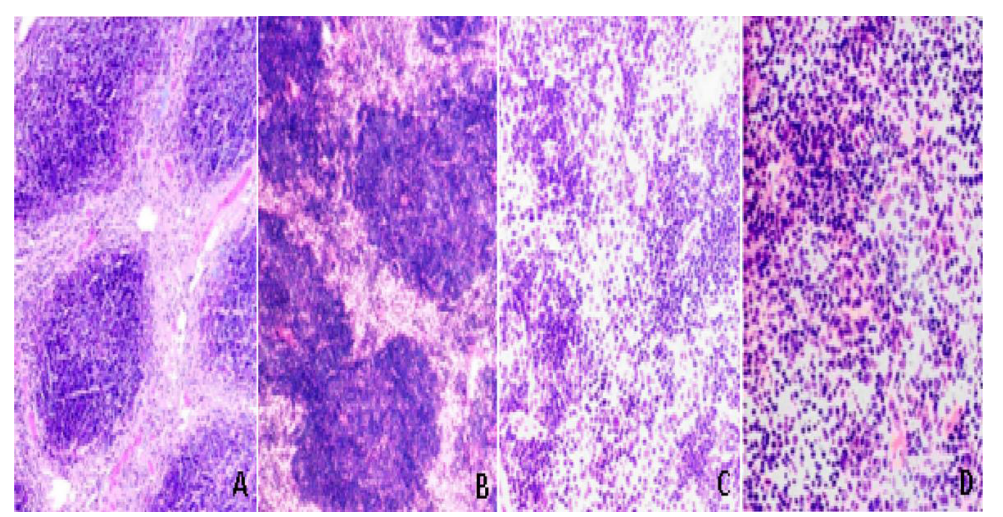

Figure 1. Histopathological changes of the spleens of C57BL/6 mice from different groups (hematoxylin and eosin; A. B. low power; C. D. middle power). 


\section{DC immunophenotype analysis}

Contents of the negative co-stimulatory molecules CD $11 \mathrm{c}^{+} \mathrm{PD}-\mathrm{L} 1^{+} \mathrm{DC}$ and $\mathrm{CD} 11 \mathrm{c}^{+}$ PD- $1^{+}$DC significantly increased in the early disease course $(6$ and $12 \mathrm{~h})(\mathrm{P}<0.05)$, and then rapidly decreased. At $24 \mathrm{~h}$ and $48 \mathrm{~h}$, their levels were restored to control group levels. After 5 days, they increased again, and continued to increase to the final stage of MODS (10-12 days) to reach the peak values $(\mathrm{P}<0.01)$. In addition, the CD11 $\mathrm{c}^{+} \mathrm{I}-\mathrm{A}^{\mathrm{b}+} \mathrm{DC}$ and $\mathrm{CD} 11 \mathrm{c}^{+} \mathrm{CD} 86^{+} \mathrm{DC}$ contents significantly increased in the early disease course $(6$ and $12 \mathrm{~h})(\mathrm{P}<0.05)$ and then began to reduce at $24 \mathrm{~h}$. At 10-12 days, the levels approached those of the control group (Table 1).

Table 1. Expressions of PD-L1, PD-1, I-Ab, and CD86 in splenic dendritic cells (\%).
\begin{tabular}{lccccccc}
\hline Item & Control group & 6-h group & 12-h group & 24-h group & 48-h group & 5-7-day group & 10-12-day group \\
\hline PD-L1 & $54.5 \pm 20.1$ & $78.4 \pm 24.5^{\#}$ & $69.0 \pm 23.9^{\#}$ & $57.4 \pm 17.3$ & $56.0 \pm 18.7$ & $62.6 \pm 21.2$ & $81.7 \pm 21.8^{*}$ \\
PD-1 & $0.6 \pm 0.2$ & $8.2 \pm 3.8^{*}$ & $6.1 \pm 3.3^{*}$ & $4.5 \pm 2.4^{\#}$ & $1.9 \pm 0.8$ & $5.2 \pm 2.18^{\#}$ & $9.9 \pm 4.2^{*}$ \\
I-A & $27.2 \pm 5.9$ & $48.0 \pm 12.9^{\#}$ & $62.6 \pm 25.2^{*}$ & $56.3 \pm 25.6^{\#}$ & $39.5 \pm 11.4$ & $40.8 \pm 15.3$ & $27.6 \pm 14.7$ \\
CD86 & $24.4 \pm 12.1$ & $69.3 \pm 28.6^{*}$ & $53.8 \pm 17.4^{*}$ & $51.6 \pm 17.9^{\#}$ & $46.2 \pm 15.3^{\#}$ & $35.9 \pm 11.7$ & $27.5 \pm 9.8$ \\
\hline
\end{tabular}

vs the control group; ${ }^{*} \mathrm{P}<0.01,{ }^{*} \mathrm{P}<0.05$.

\section{DISCUSSION}

Recent research on the pyemia-MODS prevention mechanism has revealed the involvement of immune imbalance and immunosuppression. DCs have become a research hotspot in immunology in recent years. They not only induce the body's immune response, but also participate in the induction of the body's immune tolerance system. Our previous studies showed that splenic DC overactivity in the early stage of pyemia promoted an excessive inflammatory reaction, and massive apoptosis and regression of splenic DCs and lymphocytes in the advanced stage of MODS, which was accompanied by reduced expressions of the co-stimulatory molecules CD86, CD80, and MHC-II. In addition, the number of $\mathrm{T}$ lymphocytes and interleukin (IL)-12 expression were obviously reduced. These findings indicated that immunosuppression in the advanced stage of pyemia was related to a decrease in DC activity (Li et al., 2003; Lu et al., 2006, 2007). In recent years, research on the negative immune regulation mechanism of DC has indicated that the body's immunosuppression mechanism is possibly related to the generation of a number of tolerant DC cells ( $\mathrm{Lu}$ et al., 2006; Poehlmann et al., 2009).

$\mathrm{DC}$ regulation of the $\mathrm{T}$ cell immune response is achieved by the signal transmission between DC and T cells. MHC-Ag on the DC surface binds to the T cell receptor (TCR) on the $\mathrm{T}$ cell surface to transmit the first signal, and the co-stimulatory molecules expressed by $\mathrm{DC}$ bind to the corresponding receptors on the T cell surface to generate the second signal. In addition, the MHC-II molecule expression level can reflect the functional status of DCs. The B7 family is the most important of all co-stimulatory molecules. B7-1 (CD80) and B7-2 (CD86) provide positive stimulus signals for the cell immune response (Greenwald et al., 2005). PD-1 (CD279) is expressed on the T cell surface, and mainly induces the inactivation $\mathrm{CD} 4^{+} \mathrm{T}$ cells, $\mathrm{CD} 8^{+} \mathrm{T}$ cells, natural killer (NK) T cells, B cells, monocytes, and DCs (Keir et al., 2007). PD-L1 (B7-H1, CD274) is a newly identified member of the B7 family, which is a ligand of PD-1, mainly expressing in DCs, macrophages, $\mathrm{B}$ cells, and partial T cells. 
PD-L1 and PD-1 act as negative co-stimulatory signal molecules to provide negative costimulatory signals for cellular immunity (del Rio et al., 2008; Riley, 2009). Some studies suggest that the PD-1/PD-L1 inhibition pathway participates in the body's immune tolerance caused by tumors and infection of viruses such as HBV, HCV, and HIV, and also plays an important role in implant immune tolerance (Dong and Chen, 2006; Okazaki and Honjo, 2007; Parekh et al., 2009). However, there is no report on its roles in pyemia and MODS immunosuppression.

In this study, excessive zymosan was administered by intraperitoneal injection to establish aseptic peritonitis and pyemia mouse models. At the advanced stage of the disease course (10-12 days), MODS was formed. The spleen tissue structures of test mice in the 24-, 48-h, and 10-12-day groups were obviously damaged. In addition, the contents of negative co-stimulatory molecules in DCs presented a trend of increasing-reducing-increasing throughout the MODS disease course. The PD-L1 expression of splenic DCs significantly increased in the early disease course $(6 \mathrm{~h})(78.4 \pm 34.5 \%, \mathrm{P}<0.05)$ and then rapidly reduced and restored to the normal level at 24 and $48 \mathrm{~h}$. After 5 days, PD-L1 increased again and continued to increase to the advanced stage of MODS (10-12 days) to reach the peak value $(81.7 \pm 31.8 \%, \mathrm{P}<0.01)$. The PD-1 expression level in splenic DCs was lower, but showed the same pattern of change as observed for PD-L1. The DC contents of the MHC-II molecule and the co-stimulatory molecule CD86 first increased and then reduced continuously in the disease course of MODS. The expressions of I- $\mathrm{A}^{\mathrm{b}}$ and CD86, reflecting DC activity, significantly increased in the early trauma phase $(6 \mathrm{~h})$, and then began to reduce at $24 \mathrm{~h}$, reaching the normal level at 10-12 days.

In the early stage of the disease course, after splenic DCs were stimulated by antigens, I-A $\mathrm{A}^{\mathrm{b}}$ and $\mathrm{CD} 86$ expressions rapidly increased, and splenic DCs matured to activate lymphocytes to generate the immune response; the immune activation of active DCs was predominant at this stage. At the same time, negative co-stimulatory molecules were highly expressed, indicating that while DCs are playing an immune activation role, they have a negative regulatory effect to inhibit the overexpression of immune activity. With disease progress, excessive injected zymosan caused the inflammatory reaction to continuously spread and the downregulation of $\mathrm{I}-\mathrm{A}^{\mathrm{b}}$ and CD86. Therefore, the DC immune activation function decreased. In addition, the PD-L1 and PD-1 expressions continuously increased, indicating that early active DCs had converted into tolerant DCs. In the MODS stage, the immune tolerance caused by the DCs' highly negative co-stimulatory molecules was predominant. The results of this study preliminarily suggest that DCs can regulate $\mathrm{T}$ cell function by means of different co-stimulatory molecules. In the early disease course, active DC proliferation is predominant in the spleen, whereas in the MODS stage, tolerant DCs are predominant. The expressions of co-stimulatory molecules of tolerant DCs and MHC-II molecules reduced, while negative co-stimulatory molecule expression was upregulated. DCs can inhibit T cell function by means of PD-L1 and PD-1 molecules to play a negative immune regulation role to induce immunosuppression formation resulting in MODS occurrence.

\section{ACKNOWLEDGMENTS}

Research supported by the "Eleventh Five" Medical Research Project of PLA (\#06MB307). 


\section{REFERENCES}

Brahmer JR, Tykodi SS, Chow LQ, Hwu WJ, et al. (2012). Safety and activity of anti-PD-L1 antibody in patients with advanced cancer. N. Engl. J. Med. 366: 2455-2465.

del Rio ML, Buhler L, Gibbons C, Tian J, et al. (2008). PD-1/PD-L1, PD-1/PD-L2, and other co-inhibitory signaling pathways in transplantation. Transpl. Int. 21: 1015-1028.

Dong H and Chen X (2006). Immunoregulatory role of B7-H1 in chronicity of inflammatory responses. Cell Mol. Immunol. 3: $179-187$.

Freeman GJ, Long AJ, Iwai Y, Bourque K, et al. (2000). Engagement of the PD-1 immunoinhibitory receptor by a novel B7 family member leads to negative regulation of lymphocyte activation. J. Exp. Med. 192: 1027-1034.

Greenwald RJ, Freeman GJ and Sharpe AH (2005). The B7 family revisited. Annu. Rev. Immunol. 23: 515-548.

Keir ME, Francisco LM and Sharpe AH (2007). PD-1 and its ligands in T-cell immunity. Curr. Opin. Immunol. 19: 309-314.

Keir ME, Butte MJ, Freeman GJ and Sharpe AH (2008). PD-1 and its ligands in tolerance and immunity. Annu. Rev. Immunol. 26: 677-704.

Li ZH, Lu JY, Wang XH and Yang Y (2003). Change in IL-12 expression and its role in immunodissonance in mods mice. Med. J. Chin. People's Liberation Army 28: 887.

Lu JY, Li ZH, Wang XH and Yang Y (2007). Pathologic alteration of spleen and its dendritic cells in patients with multiple organ dysfunction syndrome: An observation of 52 autopsied cases. Chin. J. Diagnostic. Pathol. 14: 172-175.

Lu JY, Li ZH, Wang XH, Yang Y, et al. (2006). Changes in spleen dendritic cells in multiple organ dysfunction syndrome in mice. Zhongguo Wei Zhong Bing Ji Jiu Yi Xue 18: 24-27.

Lyn-Kew K and Standiford TJ (2008). Immunosuppression in sepsis. Curr. Pharm. Des. 14: 1870-1881.

Nishimura H and Honjo T (2001). PD-1: an inhibitory immunoreceptor involved in peripheral tolerance. Trends Immunol. 22: $265-268$.

Okazaki T and Honjo T (2007). PD-1 and PD-1 ligands: from discovery to clinical application. Int. Immunol. 19: 813-824

Parekh VV, Lalani S, Kim S, Halder R, et al. (2009). PD-1/PD-L blockade prevents anergy induction and enhances the anti-tumor activities of glycolipid-activated invariant NKT cells. J. Immunol. 182: 2816-2826.

Peng G, Luo B, Li J, Zhao D, et al. (2011). Hepatitis B e-antigen persistency is associated with the properties of HBVspecific CD8 T cells in CHB patients. J. Clin. Immunol. 31: 195-204.

Poehlmann H, Schefold JC, Zuckermann-Becker H, Volk HD, et al. (2009). Phenotype changes and impaired function of dendritic cell subsets in patients with sepsis: a prospective observational analysis. Crit. Care 13: R119.

Riley JL (2009). PD-1 signaling in primary T cells. Immunol. Rev. 229: 114-125.

Selenko-Gebauer N, Majdic O, Szekeres A, Höfler G, et al. (2003). B7-H1 (programmed death-1 ligand) on dendritic cells is involved in the induction and maintenance of T cell anergy. J. Immunol. 170: 3637-3644.

Tseng SY, Otsuji M, Gorski K, Huang X, et al. (2001). B7-DC, a new dendritic cell molecule with potent costimulatory properties for T cells. J. Exp. Med. 193: 839-846.

Xu H, Wang X, Pahar B, Moroney-Rasmussen T, et al. (2010). Increased B7-H1 expression on dendritic cells correlates with programmed death 1 expression on $\mathrm{T}$ cells in simian immunodeficiency virus-infected macaques and may contribute to T cell dysfunction and disease progression. J. Immunol. 185: 7340-7348. 